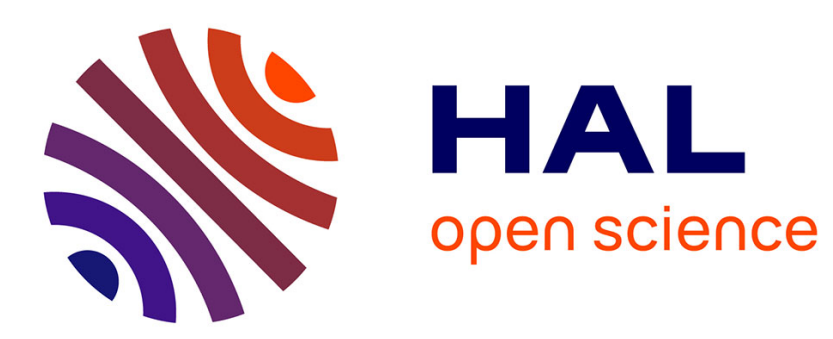

\title{
Propriétés statiques des transistors M. O. S. de puissance à canal vertical. Cas du régime de non-pincement
}

\author{
P. Rossel, H. Martinot, M. Zamorano
}

\section{- To cite this version:}

P. Rossel, H. Martinot, M. Zamorano. Propriétés statiques des transistors M. O. S. de puissance à canal vertical. Cas du régime de non-pincement. Revue de Physique Appliquée, 1978, 13 (1), pp.23-28. 10.1051/rphysap:0197800130102300 . jpa-00244417

\section{HAL Id: jpa-00244417 https://hal.science/jpa-00244417}

Submitted on 1 Jan 1978

HAL is a multi-disciplinary open access archive for the deposit and dissemination of scientific research documents, whether they are published or not. The documents may come from teaching and research institutions in France or abroad, or from public or private research centers.
L'archive ouverte pluridisciplinaire HAL, est destinée au dépôt et à la diffusion de documents scientifiques de niveau recherche, publiés ou non, émanant des établissements d'enseignement et de recherche français ou étrangers, des laboratoires publics ou privés. 


\title{
Classification \\ Physics Abstracts \\ $73.00-73.25-73.40 \mathrm{Q}$ \\ PROPRIÉTÉS STATIQUES DES TRANSISTORS M. O. S. DE PUISSANCE A CANAL VERTICAL CAS DU RÉGIME DE NON-PINCEMENT
}

\author{
P. ROSSEL, H. MARTINOT et M. ZAMORANO \\ Laboratoire d'Automatique et d'Analyse des Systèmes du C. N. R. S. \\ 7, avenue du Colonel-Roche, 31400 Toulouse, France
}

(Reçu le 8 juin 1977, accepté le 21 septembre 1977)

\begin{abstract}
Résumé. - Les propriétés électriques des transistors M. O.S. de puissance diffusés à multicanaux verticaux sont étudiées dans le cas du régime de fonctionnement non pincé. Les phénomènes pris en compte sont : la réduction de mobilité due au champ électrique, l'inhomogénéité du dopage dans le canal, l'échauffement du cristal sous l'action de la puissance continue dissipée. Il est montré d'une part que la tension de seuil est déterminée par la valeur maximale du dopage près de la source et d'autre part que le courant en région non pincée est contrôlé par le dopage au voisinage du drain et par la température du silicium. La comparaison entre les résultats expérimentaux et les valeurs théoriques est effectuée.
\end{abstract}

Abstract. - The electrical properties of verticaly diffused multi-channel power M. O. S. transistors are discussed in the not-pinched off regime. The phenomena taken into account are :

i) The reduction of mobility due to the electric field ;

ii) The channel inhomogeneous doping level ;

iii) The crystal heating by the d. c. power dissipation.

It is shown that on the one hand the threshold voltage is determined by the maximum doping level near the source diffusion and that on the other hand the current in the not pinched-off region is controlled by both the doping level near the drain diffusion and the substrate temperature. The comparison between experimental results and theoretical predictions is carried out.

Les transistors M. O.S. réalisés par double diffusion (D. M. O. S.) ont permis de diminuer les longueurs des canaux d'inversion en utilisant les processus développés pour les technologies avancées des transistors bipolaires [1], [2], [3]. Les principaux avantages de ces dispositifs sont d'une part leur tenue aux tensions élevées [4] et d'autre part leur grande vitesse de commutation. La mise au point de l'attaque anisotrope du silicium [5] a permis de réaliser des structures M. O. S. diffusées à canal vertical (V. M. O. S.) qui présentent les mêmes qualités potentielles que les dispositifs D. M. O. S. Cette configuration verticale rend possible la mise en parallèle d'un grand nombre de canaux par utilisation d'un drain commun. Ces V. M. O. S. sont particulièrement bien adaptés à l'amplification de puissance en basses et hautes fréquences [6], [7].

Dans cet article nous proposons une méthode de calcul des caractéristiques courant-tension de ces V.M. O.S. en régime de non-saturation, en tenant compte de la réduction de mobilité due aux champs électriques longitudinal et transversal, de l'échauffement du cristal sous l'action de la puissance dissipée, et de la non-uniformité du dopage dans la direction source-drain.

1. Caractéristiques à faible tension drain-source ; tension de seuil. - La figure 1 représente la coupe schématique (1a) d'un élément du transistor à canal $\mathbf{N}$

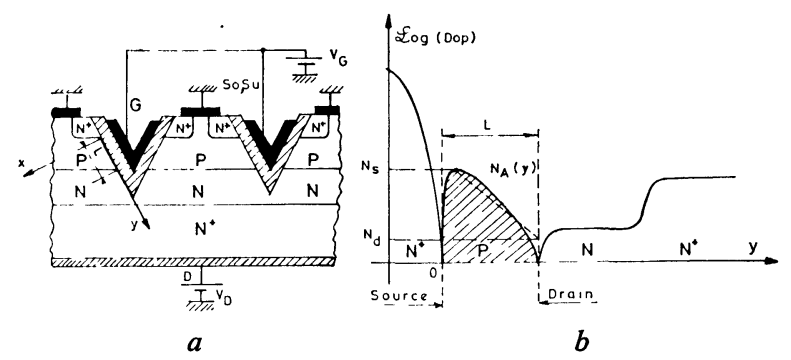

FIG. 1. - 1a) Coupe schématique de la structure. métal. rman oxyde de grille. 1 ) ) Profil de dopage dans la direction source-drain (échelles arbitraires). - . . . - approximation utilisée dans la région du canal inversée.

[1a) Cross-section of the structure. metal. oxide. $1 b$ ) Doping profile in the source drain direction (arbitrary scale) ..... approximation in the inverted channel.] 
réalisé dans une attaque en $\mathrm{V}$ anisotrope du silicium ainsi que le profil de dopage [4] dans la direction $\mathrm{O} y(1 b)$. Ce profil dans la région du canal d'inversion est imposé par les conditions de diffusion de la zone $\mathrm{P}$ et des redistributions lors de la diffusion de la source $\mathrm{N}^{+}$puis de la croissance thermique de l'oxyde de grille [8]. Il peut être défini en première approximation par la relation :

$$
N_{\mathrm{A}}(y)=N_{\mathrm{s}} \exp \frac{y}{L} \log \frac{N_{\mathrm{d}}}{N_{\mathrm{s}}}
$$

où $N_{\mathrm{s}}$ et $N_{\mathrm{d}}$ représentent les valeurs maximale au voisinage de la source et minimale extrapolée au droit du drain métallurgique.

Pour les faibles valeurs de la tension drain-source $V_{\mathrm{D}}$, le calcul du courant de drain s'effectue au moyen des étapes suivantes :

(i) Détermination de la charge totale des porteurs minoritaires $q N(\Xi, y)$ au point d'abscisse $y$ par intégration de l'équation de Poisson dans le semiconducteur suivant la direction $x$ et par écriture de la continuité de la composante normale de l'induction électrique à l'interface silice-silicium [9] :

$$
\begin{aligned}
q N(\Xi, y)=C_{0 \mathbf{x}}\{[ & \left.V_{\mathbf{G}}^{\prime}-\Xi-2 \varphi_{\mathrm{F}}(y)\right]- \\
& \left.-\varphi_{\mathbf{B}}^{1 / 2}(y)\left[\Xi+2 \varphi_{\mathrm{F}}(y)\right]^{1 / 2}\right\}
\end{aligned}
$$

où $C_{0 \mathrm{x}}$ est la capacité d'oxyde de grille par unité de surface, $\varphi_{\mathrm{F}}(y)$ le potentiel de Fermi au point d'abscisse $y, \Xi$ l'écart en potentiel des pseudo-niveaux de Fermi, $V_{\mathrm{G}}^{\prime}$ la tension effective de grille incluant l'effet des charges d'oxyde et d'interface, ramenées à l'interface, et du travail de sortie métal-semiconducteur :

$$
\begin{aligned}
\varphi_{\mathrm{F}}(y) & =\frac{k T}{q} \log \frac{N_{\mathrm{A}}(y)}{n_{\mathrm{i}}} \\
V_{\mathrm{G}}^{\prime} & =V_{\mathrm{G}}+\frac{Q_{\mathrm{ss}}}{C_{0 \mathrm{x}}}-\varphi_{\mathrm{MS}} .
\end{aligned}
$$

Le potentiel $\varphi_{B}(y)$ est défini par [10]

$$
\varphi_{\mathrm{B}}(y)=\frac{2 q \varepsilon_{\mathrm{si}} \varepsilon_{0}}{C_{0 \mathrm{x}}^{2}} N_{\mathrm{s}} \cdot \exp \left(\frac{y}{L} \log \frac{N_{\mathrm{d}}}{N_{\mathrm{s}}}\right) .
$$

(ii) Ecriture du courant qui traverse la section droite d'un élément différentiel de canal de largeur $\mathrm{d} y$ [11]

$$
I_{\mathrm{D}}=Z \cdot \mu_{\mathrm{eff}} \cdot q \cdot N(y, \Xi) \cdot \frac{\mathrm{d} \Xi}{\mathrm{d} y}
$$

où $Z$ est la largeur du canal et $\mu_{\text {ef }}$ la mobilité des porteurs affectée par le champ transversal. Cette dépendance entre mobilité effective et champ électrique transversal a été analysée par Pierret [12] et Vassilieff [13] et peut être approximée par la relation suivante :

$$
\mu_{\mathrm{eff}}=\mu_{0} \frac{1}{1+\left(V_{\mathrm{G}}^{\prime}-\Xi(y)-2 \varphi_{\mathrm{F}}(y)\right) / \psi}
$$

où $\mu_{0}$ est la mobilité à champ nul et $\psi$ un paramètre dépendant de l'épaisseur d'oxyde, du temps de relaxation et de la masse effective des porteurs à l'interface.

(iii) Intégration de l'équation différentielle (6) dans la direction source-drain. Pour ce faire, compte tenu de la dépendance logarithmique du potentiel de Fermi en fonction du dopage, les variations de ce potentiel en fonction de la coordonnée $y$ sont négligées et la valeur moyenne $\varphi_{F}$ est prise en compte

$$
\varphi_{\mathrm{F}}=\frac{\varphi_{\mathrm{F}}(y=0)+\varphi_{\mathrm{F}}(y=L)}{2} .
$$

L'erreur ainsi commise sur ce potentiel est de l'ordre du potentiel thermodynamique pour des variations d'une décade sur le dopage entre source et drain.

Pour des valeurs de la tension de drain comparables au potentiel thermodynamique $(k T / q)$ l'intégration de (6) conduit à :

$$
\begin{gathered}
I_{\mathrm{D}}=\mu_{0} \frac{Z}{L} C_{0 \mathrm{x}} \frac{V_{\mathrm{D}}}{1+\frac{V_{\mathrm{G}}^{\prime}-2 \varphi_{\mathrm{F}}}{\psi}} \frac{\left(\log \sqrt{\frac{N_{\mathrm{d}}}{N_{\mathrm{s}}}}\right) \cdot\left(V_{\mathrm{G}}^{\prime}-2 \varphi_{\mathrm{F}}\right)}{\log \left\{1+\left[\left(V_{\mathrm{G}}^{\prime}-2 \varphi_{\mathrm{F}}\right)\left(\sqrt{\frac{N_{\mathrm{d}}}{N_{\mathrm{s}}}}-1\right) /\left(V_{\mathrm{G}}^{\prime}-2 \varphi_{\mathrm{F}}-2 \varphi_{\mathrm{F}}^{1 / 2} \varphi_{\mathrm{BS}}^{1 / 2} \sqrt{\frac{N_{\mathrm{d}}}{N_{\mathrm{s}}}}\right)\right]\right\}} \\
\begin{array}{c}
\text { avec } \\
\varphi_{\mathrm{BS}}=2 q N_{\mathrm{s}} \varepsilon_{\mathrm{si}} \varepsilon_{0} / C_{0 \mathrm{x}}^{2} .
\end{array}
\end{gathered}
$$

Cette relation fait apparaître les conséquences suivantes :

(i) La tension de seuil du transistor, telle qu'elle a été définie par H. C. Pao [14] comme étant la valeur de la tension de grille qui annule la conductance de sortie à faible tension drain-source, satisfait à la condition :

$$
\left.\frac{\partial I_{\mathrm{D}}\left(V_{\mathrm{G}}\right)}{\partial V_{\mathrm{D}}}\right|_{V_{\mathrm{D}} \rightarrow 0}=0
$$

et s'exprime par :

$$
V_{\mathrm{T}}=-\frac{Q_{\mathrm{ss}}}{C_{0 \mathrm{x}}}+\varphi_{\mathrm{MS}}+2 \varphi_{\mathrm{F}}+\left(2 \varphi_{\mathrm{F}}\right)^{1 / 2} \cdot \varphi_{\mathrm{BS}}^{1 / 2}
$$

Cette expression montre d'une part que la tension de seuil est essentiellement contrôlée par la valeur du maximum de dopage $N_{\mathrm{s}}$ et est indépendante du profil de dopage dans le reste du canal et d'autre part que, compte tenu de la valeur élevée de ce dopage $N_{\mathrm{s}}$, cette 
tension de seuil dans le cas considéré des canaux $\mathrm{N}$ est, généralement positive.

(ii) Le profil de dopage étant tel que l'inégalité suivante est respectée,

$$
\frac{N_{\mathrm{d}}}{N_{\mathrm{s}}} \ll 1
$$

La conductance de sortie, pour les valeurs de la tension de grille élevées satisfaisant à l'inégalité :

$$
V_{\mathrm{G}}^{\prime}>2 \varphi_{\mathrm{F}}+\frac{2 \sqrt{2 \varphi_{\mathrm{F}} \cdot \varphi_{\mathrm{BS}}}}{\log \left(N_{\mathrm{S}} / N_{\mathrm{D}}\right)}
$$

s'exprime par :

$$
S_{0}=\left.\frac{\partial I_{D}}{\partial V_{D}}\right|_{V_{D} \rightarrow 0}=\mu_{0} \frac{Z}{L} C_{0 \times} \frac{\psi\left(V_{G}^{\prime}-2 \varphi_{F}\right)}{\psi+V_{G}^{\prime}-2 \varphi_{F}} .
$$

Par suite les variations de la quantité $\left(V_{\mathrm{G}}^{\prime}-2 \varphi_{\mathrm{F}}\right) / S_{0}$ en fonction de la tension de grille $V_{\mathrm{G}}$ sont linéaires. De ce relevé expérimental (Fig. 2) on déduit comme pour les transistors M. O. S. à dopage uniforme [13] les valeurs de paramètres $\psi$ et $\mu_{0} \frac{Z}{L} C_{0 \mathrm{x}}$.

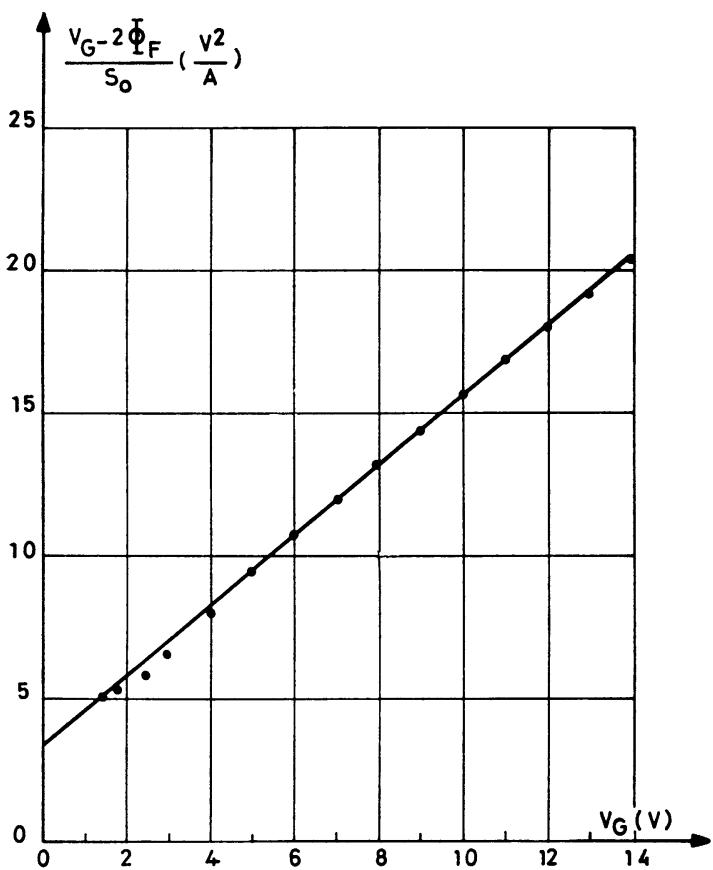

Fig. 2. - Variations expérimentales du rapport $\left(V_{\mathrm{G}}^{\prime}-2 \varphi_{\mathrm{F}}\right) / S_{0}$ en fonction de la tension de grille. Transistor V-R.

[Experimental variations of the ratio $\left(V_{\mathrm{G}}^{\prime}-2 \varphi_{\mathrm{F}}\right) / S_{0}$ as a function of the gate voltage. V-R transistor.]

On remarquera enfin que lorsque les valeurs du dopage à la source et au drain sont égales, l'expression (9) s'identifie à celle obtenue de façon classique dans le cas du dopage de canal uniforme.

2. Caractéristiques en région non saturée. 2.1 PRISE EN COMPTE DE L'EFFET DU CHAMP LONGITU-
DINAL ET DE LA TEMPÉRATURE. - Lorsque la tension de drain du transistor V. M. O. S. augmente, tout en restant inférieure à la valeur de la tension de pincement au drain [15], il est nécessaire, pour décrire les caractéristiques, de prendre en compte deux mécanismes supplémentaires qui ont été négligés dans l'analyse précédente. Il s'agit d'une part de l'effet de limitation de la vitesse des porteurs sous l'effet du champ longitudinal dans le canal, analogue à celle qui se produit dans le volume du silicium. D'autre part il faut inclure la variation des paramètres régissant le comportement électrique, sous l'effet de l'échauffement dû à la puissance continue dissipée. L'effet de réduction longitudinal de mobilité dans les couches d'inversion a été analysé par plusieurs auteurs qui ont montré que cette mobilité des porteurs peut être approchée par une loi du type [4], [13], [20] :

$$
\mu_{\mathrm{eff}}=\mu_{0} \frac{1}{\left(1+\frac{\mathrm{d} \Xi}{\mathrm{d} y} / E_{0}\right)\left[1+\left(V_{\mathrm{G}}^{\prime}-\Xi-2 \varphi_{\mathrm{F}}\right) / \psi\right]}
$$

où $E_{0}$ représente le champ critique longitudinal voisin de $2 \mathrm{~V} / \mu \mathrm{m}$. Le coefficient $\psi$ de cette relation a été introduit dans le précédent paragraphe ; il représente l'action du champ transversal. En conséquence, le courant de drain du transistor dans le cadre de l'hypothèse du canal graduel est solution de l'équation différentielle :

$$
\begin{aligned}
I_{\mathrm{D}}= & \frac{\mu_{0} Z C_{0 \mathrm{x}}}{1+\frac{\mathrm{d} \Xi}{\mathrm{d} y} / E_{0}} \cdot \frac{1}{1+\left(V_{\mathrm{G}}^{\prime}-\Xi-2 \varphi_{\mathrm{F}}\right) / \psi} \times \\
& \times\left[V_{\mathrm{G}}^{\prime}-\Xi-2 \varphi_{\mathrm{F}}-\varphi_{\mathrm{B}}^{1 / 2}(y)\left(\Xi+2 \varphi_{\mathrm{F}}\right)^{1 / 2}\right] \frac{\mathrm{d} \Xi}{\mathrm{d} y}
\end{aligned}
$$

Dans laquelle les termes $\mu_{0}, E_{0}, \psi, \varphi_{\mathrm{F}}, Q_{\mathrm{ss}} / C_{0 \mathrm{x}}, \varphi_{\mathrm{MS}}$ sont fonction de la température $T_{\mathrm{c}}$ du canal d'inversion. Si les évolutions thermiques des potentiels $\varphi_{\mathrm{F}}$ et $\varphi_{\mathrm{MS}}$ sont bien connues théoriquement, les variations des autres termes ne peuvent être obtenues en toute rigueur que par une caractérisation expérimentale suivant les méthodes développées par G. Vassilieff [13]. L'application de celles-ci au cas des structures étudiées montre que les variations des potentiels $\psi, Q_{\text {ss }} / C_{0 \mathrm{x}}$ et $\mathrm{du}$ champ $E_{0}$ peuvent être négligées dans la gamme de températures de cristal $20^{\circ} \mathrm{C}$ à $150^{\circ} \mathrm{C}$. Par contre la mobilité à champ nul décroît selon une dépendance de la forme :

$$
\mu_{0}\left(T_{\mathrm{c}}\right)=\left(\mu_{0}\right)_{0} \cdot\left(\frac{T_{\mathrm{c}}}{T_{0}}\right)^{-n}
$$

identique à celle obtenue pour les structures à dopage uniforme [13], [16], [20]. L'exposant $n$ est égal à 1,5 (Fig. 3). 


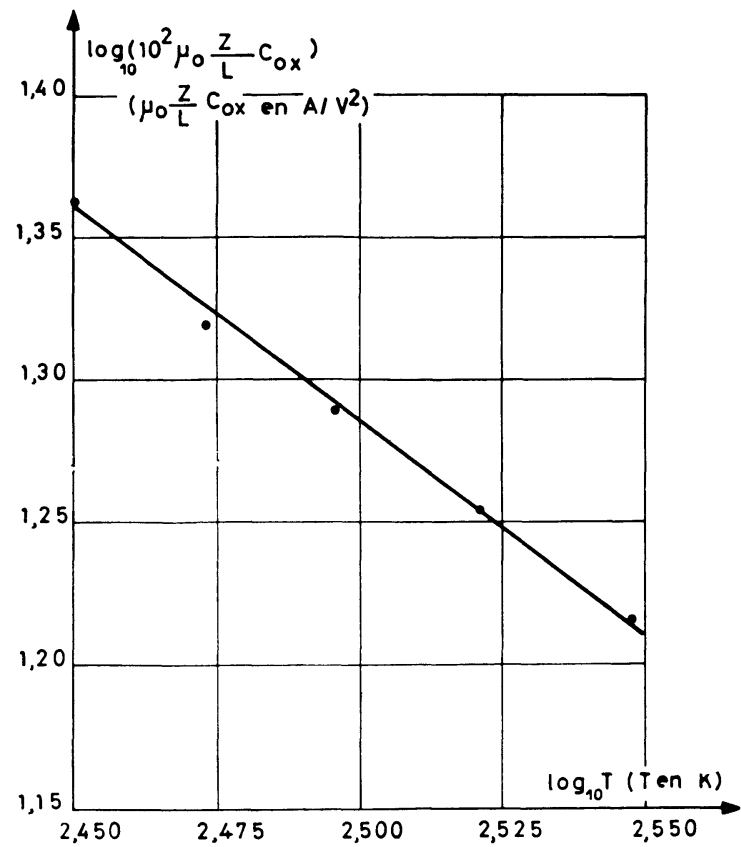

Fig. 3. - Variations en température de la mobilité à champ nul. Transistor V-P.

[Thermal variation of the low field mobility. V-P transistor.]

Par ailleurs pour un régime de fonctionnement donné, la température de cristal est régie par la relation :

$$
T_{\mathrm{c}}=T_{\mathrm{a}}+\left(R_{\mathrm{th}}+R_{\mathrm{r}}\right) V_{\mathrm{D}} \cdot I_{\mathrm{D}}
$$

Dans laquelle $T_{\mathrm{a}}$ est la température ambiante, $R_{\mathrm{th}}$ la résistance thermique cristal boîtier et $R_{\mathrm{r}}$ la résistance thermique de l'ensemble boîtier-radiateur-ambiante.

2.2 EXPRESSION DU COURANT DE DRAIN. - Le courant de drain s'exprime en intégrant l'équation différentielle (16)

$$
\begin{aligned}
I_{\mathrm{D}}= & \frac{Z C_{0 \mathrm{x}}\left(\mu_{0}\right)_{\mathrm{a}}}{L\left(1+V_{\mathrm{D}} / L E_{0}\right)} \cdot\left[\frac{T_{\mathrm{a}}+\left(R_{\mathrm{th}}+R_{\mathrm{r}}\right) V_{\mathrm{D}} \cdot I_{\mathrm{D}}}{T_{\mathrm{a}}}\right]^{-n} \times \\
& \times \int_{0}^{V_{\mathrm{D}}} \frac{\psi\left[V_{\mathrm{G}}^{\prime}-\Xi-2 \varphi_{\mathrm{F}}-\varphi_{\mathrm{B}}^{1 / 2}(y)\left[\Xi+2 \varphi_{\mathrm{F}}\right]^{1 / 2}\right]}{\psi+V_{\mathrm{G}}^{\prime}-\Xi-2 \varphi_{\mathrm{F}}} \mathrm{d} \Xi
\end{aligned}
$$

où $\left(\mu_{0}\right)_{\mathrm{a}}$ est la mobilité à champ nul à la température ambiante et $\varphi_{\mathrm{F}}$ le potentiel de Fermi moyen à la température $T_{\mathrm{a}}+\left(R_{\mathrm{th}}+R_{\mathrm{r}}\right) V_{\mathrm{D}} I_{\mathrm{D}}$. Pour les valeurs de la tension de grilles supérieures à la tension de drain, ce qui est le cas en régime de non-saturation, la quantité $\varphi_{\mathrm{B}}^{1 / 2}(y) \cdot\left(\Xi+2 \varphi_{\mathrm{F}}\right)^{1 / 2}$ au voisinage de la source est négligeable devant le potentiel $V_{\mathrm{G}}^{\prime}-\Xi-2 \varphi_{\mathrm{F}}$ puisque en ce voisinage les pseudo-niveaux de Fermi sont confondus. Par ailleurs on peut montrer par une analyse rigoureuse bidimensionnelle que ce même terme ne prend de l'importance que près du drain ou l'écart en potentiel des pseudo-niveaux de Fermi est égal à la tension de drain [18]. Par suite dans le terme sous l'intégrale figurant dans la relation (18), on peut remplacer le potentiel $\varphi_{\mathbf{B}}(y)$ par sa valeur au drain :

$$
\varphi_{\mathrm{BD}}=\frac{2 q N_{\mathrm{d}} \varepsilon_{\mathrm{si}} \varepsilon_{0}}{C_{0 \mathrm{x}^{2}}} .
$$

Le courant de drain est alors défini par la relation :

$$
\begin{aligned}
I_{\mathrm{D}}= & \frac{Z C_{0 \mathrm{x}}\left(\mu_{0}\right)_{\mathrm{a}}}{L\left(1+V_{\mathrm{D}} / L E_{0}\right)}\left[\frac{\left(T_{\mathrm{a}}+\left(R_{\mathrm{th}}+R_{\mathrm{r}}\right) V_{\mathrm{D}} I_{\mathrm{D}}\right)}{T_{\mathrm{a}}}\right]^{-n} \psi\left\{V_{\mathrm{D}}+\psi \log \frac{\psi+V_{\mathrm{G}}^{\prime}-V_{\mathrm{D}}-2 \varphi_{\mathrm{F}}}{\psi+V_{\mathrm{G}}^{\prime}-2 \varphi_{\mathrm{F}}}\right. \\
& \left.+2 \varphi_{\mathrm{BD}}^{1 / 2}\left[\left(V_{\mathrm{D}}+2 \varphi_{\mathrm{F}}\right)^{1 / 2}-\left(2 \varphi_{\mathrm{F}}\right)^{1 / 2}-\left(V_{\mathrm{G}}^{\prime}+\psi\right)^{1 / 2}\left(\operatorname{Arg} \operatorname{th} \sqrt{\frac{V_{\mathrm{D}}+2 \varphi_{\mathrm{F}}}{V_{\mathrm{G}}^{\prime}+\psi}}-\operatorname{Arg} \text { th } \sqrt{\frac{2 \varphi_{\mathrm{F}}}{V_{\mathrm{G}}^{\prime}+\psi}}\right)\right]\right\} .
\end{aligned}
$$

La conséquence immédiate de la variation de dopage dans le canal, est que le comportement électrique du transistor n'est plus symétrique contrairement aux M. O. S. T. classiques. Si l'on inverse source et drain le potentiel $\varphi_{\mathrm{BD}}$ doit être remplacé par le potentiel $\varphi_{\mathrm{BS}}$ ce qui implique que le courant sera plus faible que celui obtenu dans les conditions normales d'utilisation. Ce résultat est illustré par la figure 4 où sont tracées les variations expérimentales de la caractéristique couranttension d'un transistor à monocanal diffusé en fonctionnements normal et inverse.

2.3 COMPARAISON AVEC LES RÉSULTATS EXPÉRIMENTAUX. - Les transistors utilisés expérimentalement sont des structures à multicanaux, 128 au total, réalisés dans des $\mathrm{V}$ dont la distance inter-bord est de $6 \mu \mathrm{m}$.
La longueur $L$ de chaque canal est voisine de $2,5 \mu \mathrm{m}$ et sa largeur égale à $250 \mu \mathrm{m}$. L'aire de la puce de silicium est de $1350 \times 850 \mu \mathrm{m}^{2}$. Les valeurs des dopages $N_{\mathrm{d}}$ et $N_{\mathrm{s}}$ de la zone diffusée $\mathrm{P}$ se situent respectivement dans les gammes de quelques $10^{16}$ et $10^{15}$ atomes. $\mathrm{cm}^{-3}$. La résistivité de la région $\mathbf{N}$ épitaxiale, côté drain, est de $5 \Omega . \mathrm{cm}$ et sa longueur est inférieure à $5 \mu \mathrm{m}$.

Les paramètres du modèle $\left(\mu_{0}\right)_{\mathrm{a}} \frac{Z}{L} C_{0 \mathrm{x}}, \psi, \varphi_{\mathrm{BD}}, \varphi_{\mathrm{F}}$, $\frac{Q_{\mathrm{ss}}}{C_{0 \mathrm{x}}}-\varphi_{\mathrm{MS}}$ sont soit déterminés par la mesure des variations de la conductance de sortie en fonction de la tension de grille soit calculés à partir de la mesure de la tension de seuil. La résistance thermique $R_{\mathrm{th}}$ est obtenue par une méthode classique [19] : elle consiste à 


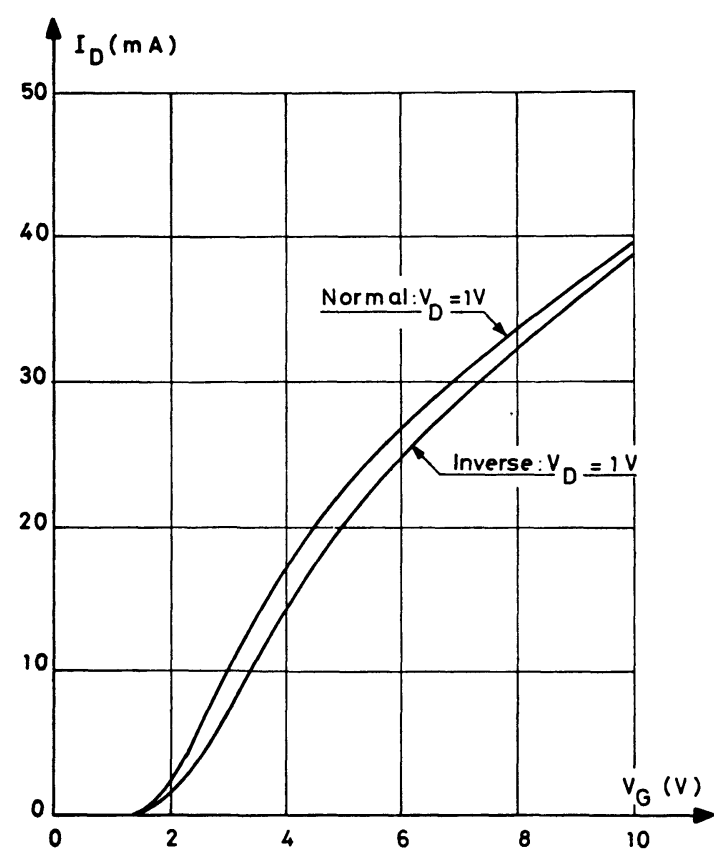

Fig. 4. - Influence de la variation du dopage sur le comportement électrique du transistor. Caractéristiques expérimentales normale et inverse d'une structure à monocanal diffusé.

[Effect of the doping profile on the electrical behaviour. Experimental characteristics ; normal and reverse configuration. Single channel diffused structure.]

déterminer l'échauffement du cristal en régime permanent à la suite de l'application d'un échelon de puissance, le transistor étant conducteur sur radiateur infini refroidi par un fluide. L'échauffement est déterminé par blocage impulsionnel au moyen de la grille et mesure du courant de la diode drain-substrat $P$. L'étalonnage préalable en température de cette caractéristique de diode fournit la valeur de cet échauffement. La figure 5 montre que le concept de résistance thermique globale $R_{\text {th }}$ constante en fonction de la puissance dissipée, est valable pour les structures étudiées.

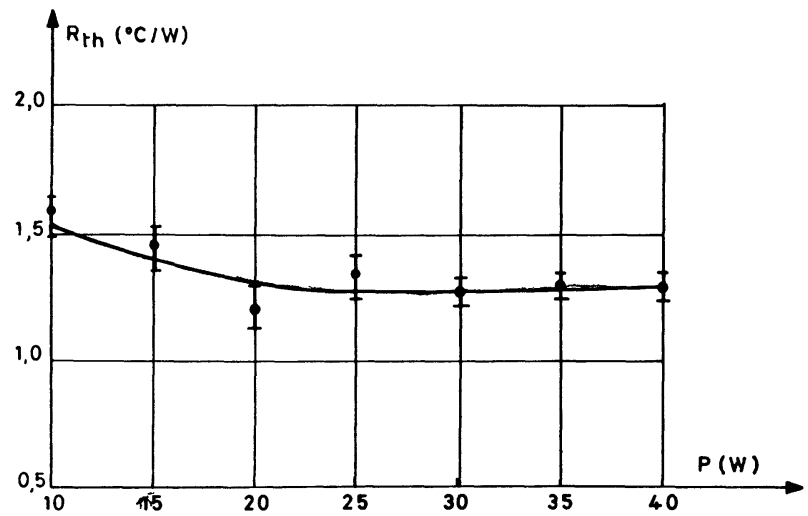

FIG. 5. - Variation de la résistance thermique cristal-boîtier avec la puissance dissipée.

[Variations of the can-chip thermal resistance as a function of the d. c. power.]
Par ailleurs la résistance thermique $R_{\mathrm{r}}$ est fixée par les caractéristiques du radiateur. Le courant de drain théorique est calculé par résolution de l'équation implicite (20). La figure 6 montre un exemple de

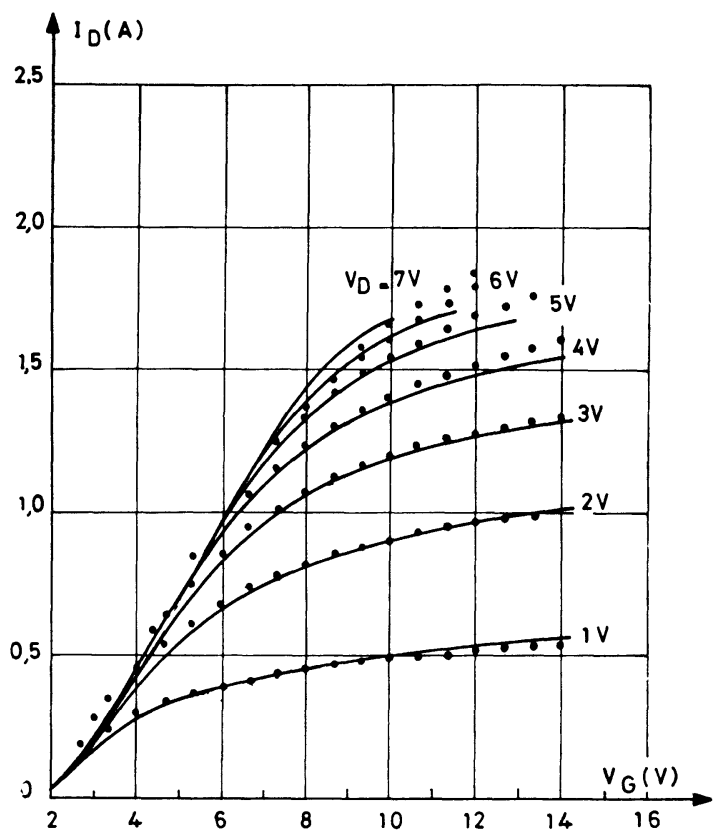

FIG. 6. - Caractéristiques statiques $I_{\mathrm{D}}\left(V_{\mathrm{G}}\right)$. Transistor V-R sur radiateur $\left(R_{\mathrm{r}}=6^{\circ} \mathrm{C} / \mathrm{W}\right)$. - expérience. $\bullet$ théorie.

[Static characteristics $I_{\mathrm{D}}\left(V_{\mathrm{G}}\right)$. V-R transistor with heat-sink $\left(R_{\mathrm{r}}=6^{\circ} \mathrm{C} / \mathrm{W}\right) .-$ experiments. $\bullet$ theory.]

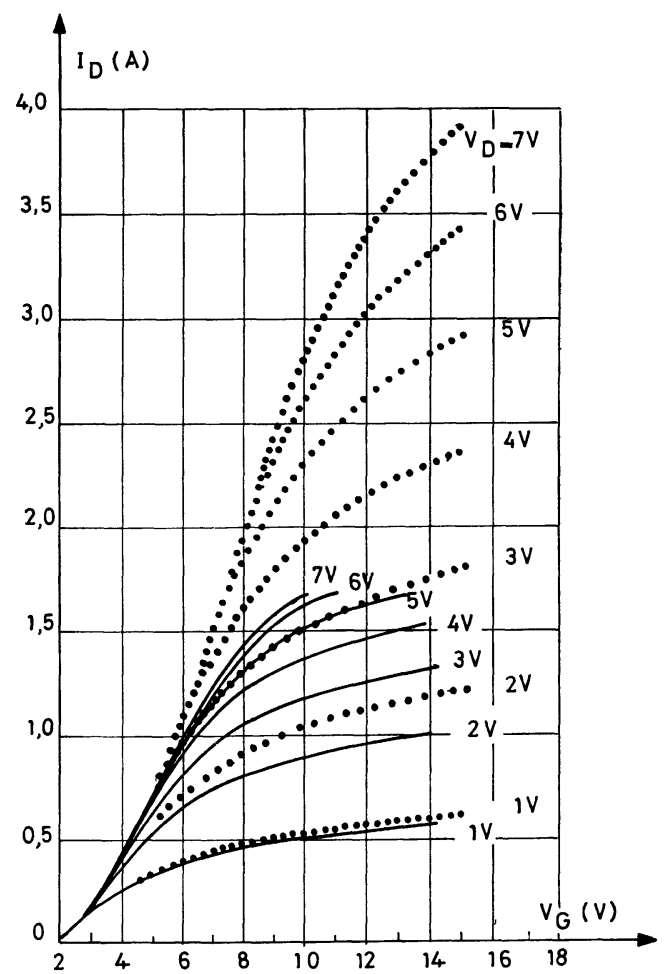

Fig. 7. - Comparaison entre caractéristiques expérimentales et théoriques (les effets thermiques ne sont pas pris en compte). Transistor V-R. - expérience, • théorie.

[Comparison between the experimental characteristics and the theoretical ones if neglecting thermal effects. V-R transistor. - experiments. • theory.] 
comparaison entre la simulation et le relevé des caractéristiques expérimentales. Il apparaît que pour les valeurs élevées de la tension de grille l'expression proposée rend compte du comportement expérimental. Pour les valeurs faibles de la tension de drain, et pour des tensions de grille voisines de la tension de seuil, des écarts apparaissent; ils doivent être attribués à l'effet du dopage au droit de la source qui comme nous l'avons déjà indiqué régit la conduction près de la tension de seuil. Enfin, les courbes théoriques ont été limitées par la valeur de tension grille qui conditionne l'annulation de la charge totale des porteurs minoritaires au droit du drain [15] ; cette hypothèse simplificatrice ne permet pas de décrire les propriétés du transistor en régime de saturation. Pour terminer, on a tracé sur la figure 7 les caractéristiques théoriques obtenues en négligeant tout effet thermique. Il apparaît que les valeurs du courant ainsi déterminé sont trop élevées et justifient la nécessité de prendre en compte l'échauffement du cristal.

3. Conclusion. - De cette étude relative à l'établissement du modèle de la conduction dans les transistors M.O.S. à canal vertical diffusé, on retiendra d'une part que la tension de grille de seuil, est essentiellement contrôlée par la région de canal la plus dopée, au voisinage de la source. Par ailleurs la non-constance du dopage dans le canal d'inversion se traduit par une dissymétrie des caractéristiques obtenues en inversant drain et source, ce qui n'est pas le cas dans les transistors M. O. S. classiques. Enfin la conduction en régime de non-saturation est essentiellement contrôlée par la valeur du dopage au droit du drain et est affectée par l'effet d'échauffement du cristal dû à la forte densité locale de puissance.

Pour ce qui est de la réduction de mobilité sous l'effet du champ électrique, on a pu observer que les lois régissant ce phénomène dans le cas des transistors à canal uniforme sont applicables aux structures diffusées.

Ainsi, les transistors M. O. S. à canal vertical dont le principal avantage structurel est lié à la mise en parallèle simple de plusieurs canaux courts présentent électriquement à la fois l'intérêt des transistors horizontaux à fort dopage de canal - de par la valeur positive du seuil - et l'intérêt des mêmes composants horizontaux à faible dopage - de par l'effet prépondérant du dopage au droit du drain sur la valeur du courant.

\section{Bibliographie}

[1] Sigg, H. J., Vandelin, G. D., Cauger, T. P., Kocsis, J. D., M. O.S. Transistor for microware applications. IEEE Trans. Electron. Devices 19 (1972).

[2] Rogers, T. L., Asai, S., Pocha, M. D., Dutton, R. W., MeINDL, J. D., An experimental an theoretical analysis of double diffused M. O. S. transistors. IEEE J. SolidState Circuits 10 (1975) 322.

[3] Pocha, M. D., Dutton, R. W., A. C. A. D. model for high voltage D. M. O. S. transistor. IEEE J. Solid-State Circuits 11 (1976) 718.

[4] Pосна, M. D., High voltage double diffused M. O. S. transistors for integrated circuits. Ph. D. Thèse, Stanford University, March 1976.

[5] Yu, S. Y., OU-YaNG, P., New V Groove double diffused M. O. S. (V. M. O. S.), Electron. Lett. 12 (1976) 605.

[6] Koor, M. V., RaGle, L., M. O. S. moves into higher power applications. Electronics 13 (1976) 98.

[7] Oakes, J. G., Wickstrom, R. A., Tremere, D. A., Heng, T. M. S., A power silicon microwave M. O. S. Transistor, IEEE Trans. Microwave Theory Tech. 24 (1976) 305.

[8] YANG, P. O., Double Ion implanted V. M. O. S. technology, IEEE J. Solid-State Circuits 12 (1977) 3.

[9] Rossel, P., Influence de la réduction de mobilité due au champ transversal sur les caractéristiques des transistors M. O. S., Electron. Lett. 5 (1969) 604.

[10] Grove, A. S., Physics and technology of semiconductor devices, Chap. 11 (John Wiley and Sons), New York, 1967.
[11] SAH, C. T., PAO, H. C., Effects of diffusion current on characteristics of metal-oxyde semiconductor transistors. Solid-State Electron. 9 (1966) 927.

[12] Pierret, R. F., Effective mobility in silicon surface channels as measured with the M. O. S. transistor. Ph. D. thèse, University of Illinois, Aug. 1966.

[13] VassiliefF, G., Modèle du transitor M. O. S. Influence des variations de la mobilité des porteurs. Thèse de $3^{\mathrm{e}}$ cycle, Université Paul Sabatier, octobre 1971.

[14] PAO, H. C., Theoretical and experimental investigations of metal-oxyde semiconductor transistors, $\mathrm{Ph}$. $\mathrm{D}$. Thèse, University of Illinois, Febr. 1966.

[15] Rossel, P., Martinot, H., VassiliefF, G., Accurate two section model for M. O.S. transistor in saturation. Solid-State Electron. 19 (1976) 51.

[16] De Graaf, H. G., van Nielen, J. A., Température influence on the channel conductance of M. O.S. transistors. Electron. Lett. 3 (1967) 195.

[17] HeydemanN, M., Résolution des équations bidimensionnelles de transport dans les semiconducteurs, Thèse de Docteur Ingénieur, Université de Paris Sud Orsay, 1972.

[18] SaINTot, P., Analyse bidimensionnelle du transistor M. O. S., Thèse de $3^{\mathrm{e}}$ cycle, Université Scientifique et Médicale de Grenoble, 1973.

[19] WalshaK, L. G., Poole, W. E., Thermal resistance measurement by I. R. Scanning, Microwave J. 2 (1977) 62.

[20] Akers, L., The analysis of the steady state thermal and electrical behavior of a Si-MOS. FET, Ph. D. Thèse, University of Texas, Aug. 1975. 